\title{
Time lapse liquid phase scanning transmission electron microscopy of nanoparticles
}

Niels de Jonge ${ }^{1,2}$, Andreas Verch, Justus Hermannsdörfer, Krijn P. de Jong ${ }^{3}$ and Jovana Zečevic ${ }^{3}$

1. INM - Leibniz Institute for New Materials, 66123 Saarbrücken, Germany.

2. Department of Physics, University of Saarland, 66123 Saarbrücken, Germany.

3. Department of Inorganic Chemistry and Catalysis, Utrecht University, Utrecht, The Netherlands.

Liquid-phase scanning transmission electron microscopy (STEM) is capable of dynamic imaging of processes taking place in liquid at the nanoscale [1]. Examples of such processes are the growth metallic nanoparticles, sintering of nanoparticles, dissolution of nanomaterials in liquid, structural changes of nanomaterials, nanoscale movements in liquid, and self-assembly processes of materials in liquid. The experimental setup typically consists of a liquid cell enclosed between thin and electron transparent windows placed in the vacuum of the electron microscopy. Both closed cells and cells connected to a microfluidic system capable of exchanging the liquid have been reported. Published liquid-phase STEM studies involved a rather wide range of sometimes unexpected phenomena occurring in the liquid. The most important factor is the interaction of the electron beam with the water causing the formation of radiolysis species such as $\mathrm{e}_{\mathrm{aq}}^{-}, \mathrm{H}^{*}, \mathrm{OH}^{*}, \mathrm{H}_{2}, \mathrm{H}_{2} \mathrm{O}_{2}, \mathrm{H}^{+}, \mathrm{OH}^{-}$. These species locally change the chemistry of the liquid enclosed within the cell, triggering reactions such as reduction of metal ions. The growth of metallic nanoparticles from the solutions can thus be initiated [2], nanoparticles may instead dissolve [3]. The scanning electron beam of STEM can even be used to induce deposition of nanoscale structures in liquid [4] and change the shapes of nanomaterials in an anisotropic manner [5], so that arbitrary patterning of nanomaterials is possible in principle. When conducting experiments in liquid it is thus crucial to understand the electrochemical effects taking place. Experiments should be designed in such way that the parameters under observation can be extracted from the observed manifold of phenomena.

A further observation was that nanoparticles moved in the liquid three orders of magnitude slower than what would be expected based on Brownian motion, if certain experimental conditions were maintained [6]. The observed slow movement was induced by surface charge on the membranes enclosing the liquid cell possibly leading to the presence of an ordered liquid layer exhibiting a viscosity five orders of magnitude larger than a bulk liquid. The degree of viscosity increase associated with such an order liquid layer is consistent with the observed dramatic slowdown of the movement. The possibility of exceptional slow motion is a key aspect of electron microscopy of specimens in liquid enabling a direct observation of the movement and agglomeration of nanoscale objects in liquid.

An overview will be given of the available systems for time-lapse liquid-phase STEM, the different observed phenomena will be presented, and it will be discussed how the temporal resolution can be improved in future.

\section{References:}

[1] N de Jonge and F M Ross, Nat Nanotechnol 6 (2011) p. 695.

[2] T Kraus and N de Jonge, Langmuir 29 (2013) p. 8427.

[3] J Hermannsdoerfer, N de Jonge and A Verch, Chem Comm 51 (2015) p. 16393.

[4] M W van de Put, et al., Small 11 (2015) p. 585.

[5] J Zecevic, et al., Small 13 (2017) p.

[6] A Verch, M Pfaff and N De Jonge, Langmuir 31 (2015) p. 6956.

[7] The authors thank E. Arzt for his support through INM. 


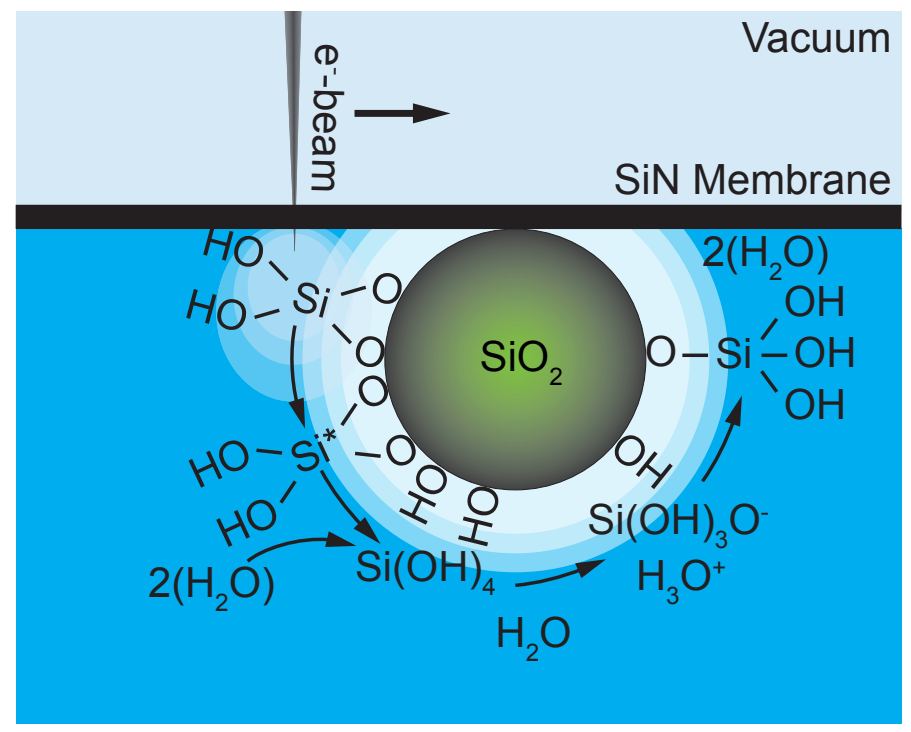

Figure 1. Schematic representation of the proposed mechanism responsible for the elongation of the silica nanoparticles. A focused electron beam scans over a sample in the indicated direction. The sample is water enclosed between silicon nitride membranes containing silica nanoparticles. The electron beam reactions with the surface silicon atoms so that dissolved $\mathrm{Si}(\mathrm{OH})_{4}$ is formed. Created silicic acid further dissociates to silanolate anion, $\mathrm{Si}(\mathrm{OH})_{3} \mathrm{O}^{-}$of which some deposits on the nanoparticle. Reprinted from [5] with permission, copyright 2017 John Wiley and Sons.

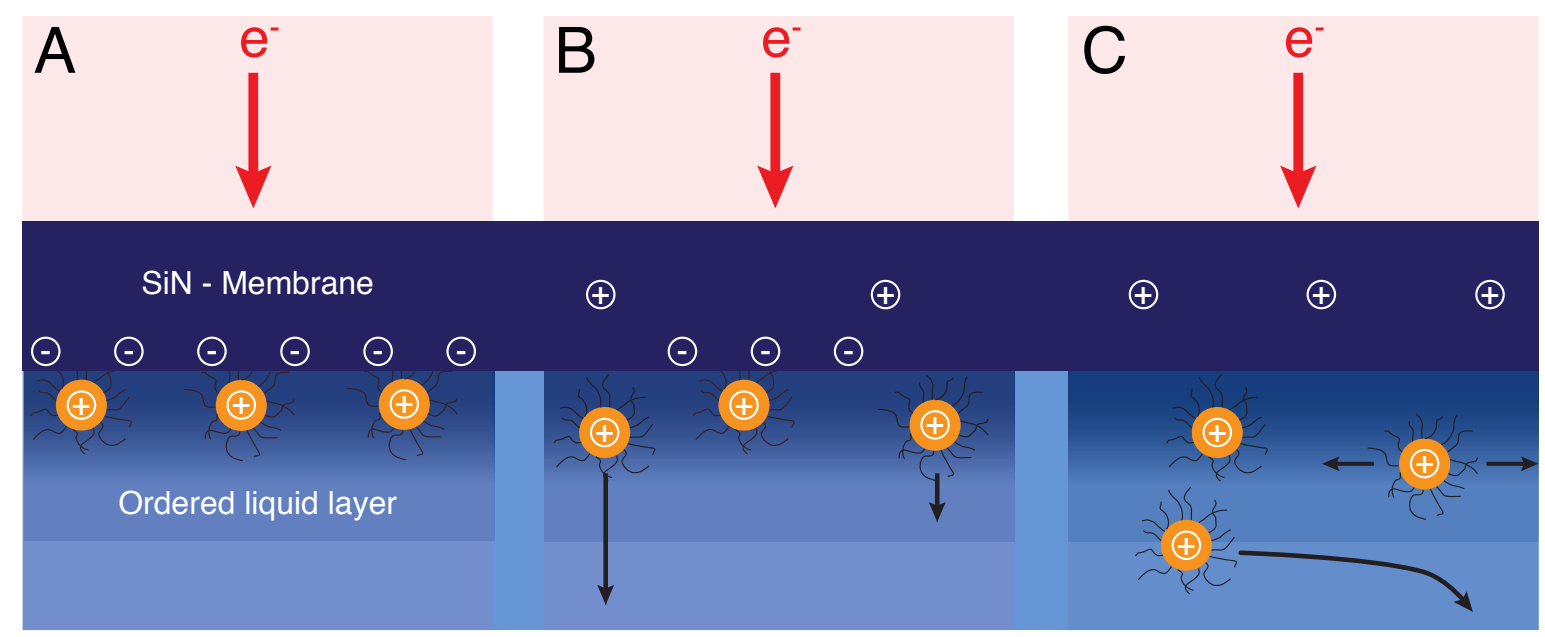

Figure 2. Model of the interaction of gold nanoparticles in liquid upon electron beam irradiation. (A) Negatively charged TCHIT coated gold nanoparticles adhered to the SiN membrane initially on account of the negative surface charge of the membrane. The first nanometers of liquid consisted of an ordered liquid layer. (B) The SiN membrane charged positively upon electron beam irradiation, so that the nanoparticles detached from the membrane. (C) Several nanoparticles moved parallel to the membrane within the ordered liquid layer at a distance in which the attractive van der Waals force was in balance with the electrostatic repulsive force. Some nanoparticles moved out of the ordered layer and started to move quickly. Reprinted with permission from [6], copyright (2015) American Chemical Society. 